\title{
Enhancing Power Flow with Dynamic Line Rating Effect Using Model Predictive Control
}

\author{
Abdelrahman Sobhy ${ }^{1,2}$, Tamer F. Megahed ${ }^{1,3}$ and Mohamed Abo-Zahhad ${ }^{1,4}$ \\ ${ }^{1}$ Egypt-Japan University of Science and Technology (E-JUST), Egypt; \\ ${ }^{2}$ MS.C students, Energy Resources Engineering Department at E-JUST; (E-mail: abdelrahman.sobhy@ejust.edu.eg) \\ ${ }^{3}$ Assistant Professor, Electrical Power Engineering at E-Just; and Electrical Engineering Department, Mansoura University, \\ Mansoura, Egypt (E-mail: tamer.megahed@ejust.edu.eg) \\ ${ }^{4}$ Professor, Dean School of Electronics, Communications and Computer Engineering at E-Just; and Department of \\ Electrical and Electronics Engineering, Assiut University, Assiut, Egypt (E-mail: mohammed.zahhad@ @just.edu.eg)
}

\begin{abstract}
Due to the high penetration of renewable energy "solar and wind", the overhead transmission line (OHTL) overloading problem arises, consequently the uncertainty increases. This makes it difficult to operate the energy system without violating the OHTL ampacity rating. To solve this problem, new transmission lines are created, which are costly and require considerable time. In the power system operation, the OHTL ampacity rating is maintained constant for all year, to ensure the safety and security of operation. However, OHTL is not used efficiently most of the time. Therefore, the dynamic line rating (DLR) is presented to ensure better utilization of available assets and to postpone the need to build new infrastructure. This paper introduces the power flow by considering the DLR characteristics of OHTL based on changes in the line ampacity due to the high penetration of intermittent sources. This paper provides a predictive control model to combine the effect of the expected DLR with the energy flow. The artificial neural network (ANN) is introduced for forecasting the DLR as a function of the conductor temperature, ambient temperature, wind speed, and solar radiation. Model predictive control (MPC) is used to control energy flow and DRL is used as a constraint in the control technique. The main features of MPC are the ability to reduce the disturbance and simple controls.
\end{abstract}

Key words. Renewable energy, overhead transmission line, dynamic line rating, model predictive control, artificial neural network

\section{Introduction}

Power system security requirements impel the system operators to limit their system components loading below its thermal limits. Transmission lines being the most exposed component to weather and environmental conditions, its ratings have been conservatively determined to avoid line deformation or stability problems. Overhead transmission line (OHTL) thermal rating is usually determined for different seasons based on the worst weather conditions. The line rating is kept constant for the entire season, which is known as the static line rating (SLR). Being dependent on the worst weather conditions, the staticline rating ensures safety and security. However, due to conservative assumptions, the line is not used efficiently most of the time. Therefore, the best way to improve the use of OHTL and delay the need to install new infrastructure is to use dynamic line rating (DLR), which aims to correlate between the OHTL ampacity with the surrounding weather conditions.

Several researchers have discussed the impact of DLR on the power system. In [1] the authors discussed the effect of ambient conditions, especially the wind speed of the OHTL cooling, and how it affects the transmission line ampacity. A statistical partial least square model for the thermal behaviors of transmission lines based on lab tests and field measurements was proposed in [2]. This model showed very good accuracy when compared to a physical model, in addition to being linear, straight forward and easier to implement. Authors in [3] presented that the high frequency of line rating changes can have negative impacts on the lifetime of an aluminum cable steel reinforced conductor. Also, they introduced the problem of choosing a reasonable frequency of rating changes so that the line can be utilized to the maximum limit without any substantial effect on the life expectancy of the line. Authors in [4] presented a method to reduce the reserve power costs which is caused by the uncertainty of intermittent renewable sources by the implementation of DLR in the dispatch system. Authors in [5] showed that DLR could increase area-to-area transmission capacity thereby impacting electricity prices and benefiting electricity consumers. Authors in [6, 7] illustrated an increase in the utilization of wind power owing to the increased load ability of a transmission line connected to a wind farm. In addition, [7] demonstrated that more wind power can be integrated into the grid with reduced load and intermittent renewable energy generation curtailments, also introduced other potential benefits of DLR including emergency control and congestion management are demonstrated.

The previous works on DLR focused mainly on wind intensive areas and considered DLR effect on accommodating more power from the wind. However, large scale PV projects have been and are being installed in Egypt. The case of DLR dependence on PV generated power is different than in the case of wind. In case of wind 
power, the line rating mostly increases with the increase in power generated from wind, while the line rating is expected to be lowered as the power from PV increases.

To achieve the best benefit from the renewable energies, considering the DLR, it is necessary to use a good control system and achieve optimal power flow. In this paper model predictive control (MPC) is used to management the power flow in the electrical grid. The main advantage of MPC makes it superior to other techniques, is that it can work in real-time, it's systematic handling of constraints, handling of dynamic multivariable systems, and including feedforward to make good use of future target information. In addition, MPC incorporates the control technique with the prediction concept, thus, MPC can anticipate the output, therefore control can be performed without disturbance. Many authors disused MPC as in [8,9] presented MPC approaches for maintaining voltage stability and to limit the severity of conductor temperature rise under contingencies in electric power systems. MPC is found to significantly reduce the solution time of the controller, allowing real-time implementation. However, these studies didn't discuss the case of high renewable energy penetration or the effect of implementing DLR.

To ensure better utilization of the grid assets and management of energy in power flow, this paper introduces an artificial neural network (ANN) for forecasting the DLR. The introduced DLR forecasting method provides the operator with the actual capability of the OHTL to carry power at any given time. With the contradictory effects of wind and PV on DLR, new means to improve energy flow performance and to correctly determine the DLR is highly required. This paper provides a predictive control model to combine the effect of the expected DLR with the energy flow.

Finally, to achieve DLR entails equipping the electrical grid by power flow sensors this leads to:-

1. Relaxing congestions due to increasing load.

2. Improving economic dispatch scenarios in contingencies.

3. Integrating renewable/distributed energy sources without grid reinforcement.

4. Deferring or avoiding uprating of OHTL ampacity.

5. Maximizing the use of alternate lines when main corridors are undergoing maintenance.

The rest of the paper is organized as follows: Section 2 introduces the DLR approaches, the factors effect of DLR, and the DLR forecasting model; Section 3 introduces the combination between the power flow and DLR; Section 4 introduces the proposed control strategy using MPC; finally, Section 5 illustrates case studies. The system under study is the south portion of the Egyptian grid which contains renewable sources such as "wind and PV". The DLR will be studied on three OHTL, the first OHTL is cutting the network crosswise with $200 \mathrm{KM}$ length, and the other two OHTL are cutting the grid lengthwise with 900 KM length.

\section{Dynamic Line Rating}

Increasing power transmission capacity requires construction of new transmission facilities costing huge amounts of money and intensive planning efforts. Thus, creating the need for a new method such as maximizing the utilization of existing assets by basing actual ampacity on actual conditions rather than the regular static rating. DLR can facilitate the accommodation of renewable energy generation in a cost-effective manner compared to other options. Compared to traditional static rating, DLR offers a more accurate rating, taking various weather factors into account. In particular, high wind speeds provide extra line cooling and ampacity, which coincides with when additional capacity is needed to accommodate higher wind generation. Even in less favorable weather conditions, dynamic line rating also protects conductors from overheating.

There are many approaches to implement the DLR such as:

1- Temperature focused approach, which monitors the conductor temperature directly, and then controls the line current so that it doesn't exceed the maximum allowable limit [10].

2- Sag focused approach, in this approach the line rating is continuously set to prevent the sag of the overhead lines is kept under safe limits [7].

3- Weather-based approach, which calculates the ampacity using loading and weather data, such as ambient temperature, wind speed, wind attack angle and solar radiation and line current [6].

In this paper weather-based approach is used to compute the DLR. During operation, a line rating model will be used to calculate the real time ampacity based on the weather forecast. The real time rating is then used in the economic dispatch.

DLR is calculated so that the conductor steady-state temperature does not exceed the maximum allowable temperature for the conductor. Steady state temperature of a conductor results from a heat balance between the heat gained and lost.

\subsection{DLR Factors}

The main factors that affect the overhead transmission line rating are solar heating depending on solar radiation, Heat transfer due to the difference between conductor and ambient temperatures, convective cooling influenced by wind speed and direction and the joule heating due to electrical loading. These variables include wind speed, wind direction, ambient temperature, electrical current and solar radiation.

1- Wind speed; Wind speed has a prevailing impact on power line ampacity as it is the main variable responsible for cooling down the conductor and hence for increasing its ampacity.

2- Wind direction; Wind angle is the angle between the wind vector and the conductor axis, which slightly changes the effective cooling of wind speed.

3- Ambient temperature; Heat transfer due to the difference between conductor and ambient air temperatures has a significant impact on line ampacity.

4- Solar radiation; Absorption of solar radiation can raise the temperature of the conductor far above the air temperature.

Fig. 1 and Fig. 2 illustrate the relation between line ampacity and wind speed, wind angle, and ambient temperature for $500 \mathrm{KV}$ line. 


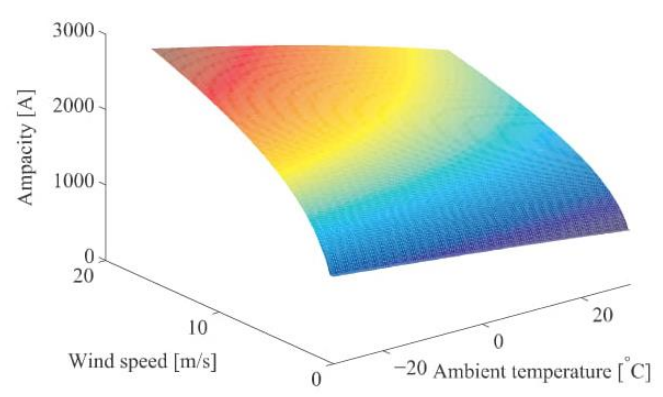

Fig. 1: relation between line ampacity and wind speed, and ambient temperature

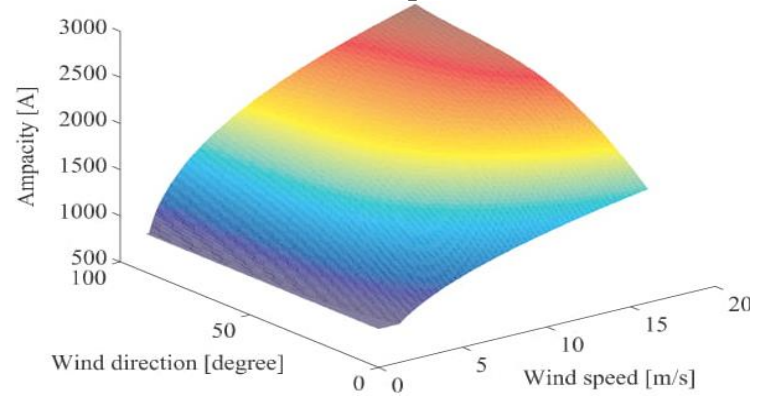

Fig. 2: relation between line ampacity and wind speed, and wind angle

Also, Fig. 3 illustrates a comparison between SLR and DLR based on the weather data. It is noticed that, DLR allows for more current to be transmitted through the OHTL most of the time.

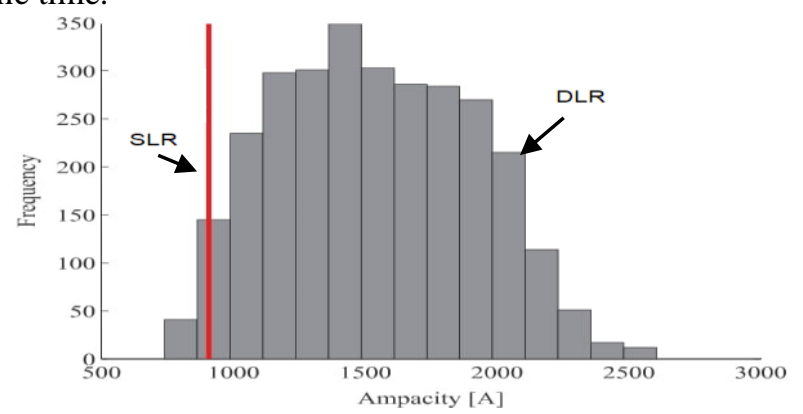

Fig. 3: DLR vs. SLR

\subsection{DLR Forecasting}

The ampacity of the OHTL is determined by the amount of current that can be safely transmitted through it, which is inversely proportional to the conductor temperature. To achieve the maximum benefit from the OHTL loading, the line temperature should be predicted in order to determine the DLR. In this paper ANN for predicting OHTL temperature, specifically, ANN is used to forecast the environmental factors "ambient temperature, wind speed, solar radiation, and the conductors current". ANN based forecasting methods that are based on simple mathematical models of the brain. Also, ANN allows complex nonlinear relationships between the response variable and its predictors. The forecasted maximum allowable current can flow in the OHTL can be deduced from the Eq. 1.

$I^{D}=\sqrt{\begin{array}{c}50302907.35-721636.2 \mathrm{~T}_{\mathrm{a}}(t) \\ +87085.9 \mathrm{~V}_{\mathrm{w}}(t-1)+36781.6 \mathrm{~V}_{\mathrm{w}} \sin (\theta)(t) \\ +33062.9 \mathrm{~V}_{\mathrm{w}} \sin (\theta)(t-1)-2850.6 \mathrm{~S}_{\mathrm{R}}(t) \\ -2645 \mathrm{~S}_{\mathrm{R}}(t-1)-1607 \mathrm{~S}_{\mathrm{R}}(t-2)\end{array}}$

Where: $I^{D}$ is the line ampacity using DLR; $T_{C}$ is the conductor temperature; $T_{a}$ is the ambient temperature; $V_{w}$ is the wind speed; $\theta$ is the wind angle; $S R$ is the solar radiation; and I is the conductor current; and the notation $(t, t-1, t-2)$ means three terms at the three consecutive instances $t, t-1$, and $t-2$.

\section{Power Flow with DLR}

The main objective of the power system is to supply the load demand with low cost and high efficiency. With the upcoming increasing the penetration of renewable energy sources and continuous increasing in electricity demand, a problem arises. Where the production of electrical energy used to be adjusted to the demand of its customers, this is not possible for most sustainable energy sources also most renewable energies are generated from locations far from loads. Instead of controlling the generation of electricity and the difficulties associated with new OHTL constructions, this paper investigates controlling the power flow using the model predictive controller based on the real-time capability in terms of OHTL ampacity.

The objective of power flow is to optimize the steady-state power system performance by increasing renewable power penetration and control the line rating by using the DLR, as shown in Eq. 2, subject to equality constraints and inequality constraints as shown in the following equations: Minimize $\quad J(x, u)$

Subject to $\quad \mathrm{g}(\mathrm{x}, \mathrm{u})=0$

$$
\mathrm{H}(\mathrm{x}, \mathrm{u}) \leq 0
$$

Where: $x$ is the dependent variables vector; and $u$ is the independent variables vector.

Equality constraints as in Eq. 5 aim to balance between load and generation. These limitations are usually called energy balance equations, which are calculated to conserve energy on each bus.

$$
\left.\begin{array}{l}
P_{b}^{G}-P_{b}^{D}=v^{*} \phi_{b} v \\
Q_{b}^{G}-Q_{b}^{D}=v^{*} \psi_{b} v
\end{array}\right\}
$$

Where: $b$ is the bus number; $P_{b}^{G}, P_{b}^{D}, Q_{b}^{G}$, and $Q_{b}^{D}$ are the active and reactive power (generation and demand); $v$ is the bus voltage; and $\phi_{b}$ and $\psi_{b}$ are Hermitian matrices. Inequality constraints give limits to bus and line ampacity. These limits are power generation limits, bus voltage limits and line rating limits. The power generation is bounded by the upper and lower limits as in Eq. 6 . The bus voltage is constrained to be in acceptable limit by the engineering limits of the network as in Eq. 7.

$$
\left.\begin{array}{l}
P_{b}^{G, \text { min }} \leq P_{b}^{G} \leq P_{b}^{G, \text { max }} \\
Q_{b}^{G, \text { min }} \leq Q_{b}^{G} \leq Q_{b}^{G, \max } \\
v_{b}^{\text {min }} \leq v_{b} \leq v_{b}^{\max }
\end{array}\right\}
$$

The main objective of line flow constraint is to achieve various goals such as avoiding line overheating; guaranteeing the stability of the network and increasing renewable power penetration instead off create OHTL. Usually, the SLR is used to be the maximum load of the OHTL; this is not fair because when using the DLR as mentioned in the previous section, the maximum utilization of OHTL loading will be achieved. Therefore; the line flow constraint as a function in DLR is shown in the following equation.

$S_{s r}=\left|v_{s}\left(v_{s}^{*}-v_{r}^{*}\right) v_{l}^{*}\right| \leq v_{s} \cdot I_{s r}^{D}$

$\Delta v_{s r}=\left|v_{s}-v_{r}\right| \leq \operatorname{amp}\left(z_{s r} I_{s r}^{D}\right)$

Where; $S_{s r}$ is the apparent power flow through the OHTL; $\boldsymbol{v}_{\boldsymbol{s}}$ and $\boldsymbol{v}_{\boldsymbol{r}}$ are the sending and receiving voltage; $\Delta \boldsymbol{v}_{\boldsymbol{s} r}$ is 
the maximum difference voltage between the two buses; and $\mathbf{z}_{\boldsymbol{s r}}$ is the OHTL impedance.

\section{Model Predictive Control}

In this paper MPC is used to manage the power flow in the electrical grid with high penetration of wind and PV power [11]. The main objective of the control is to control the flow of the OHTL according to the DLR constrain. Fig. 4 presented the control loop of MPC with ANN tools to estimate the DLR.

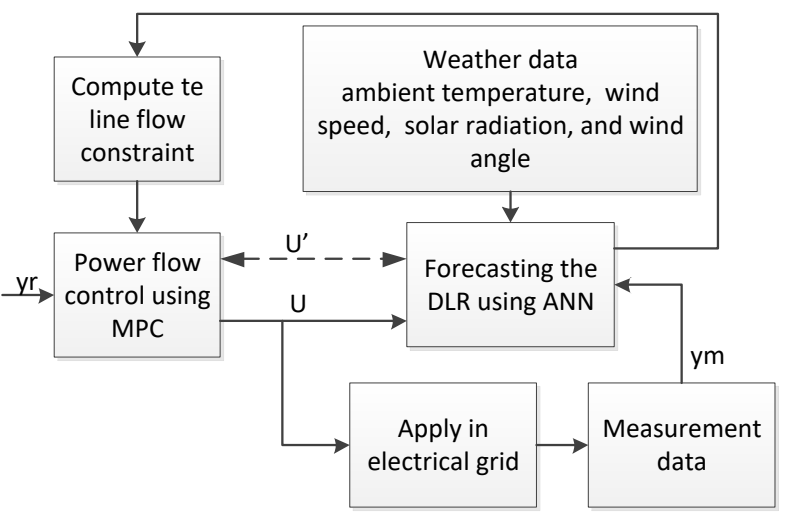

Fig. 4: control loop of MPC

MPC scheme contains five steps these steps are:

First step is the process and disturbance model where the control model predicts the expected process output behavior in the future based on the inputs and known disturbances applied to the process in the past. The following state-space description is used in this step.

$$
\left.\begin{array}{rl}
x_{t+1} & =A x_{t}+B u_{t} \\
y_{k} & =C x_{t}+d_{t} \\
z_{t} & =C x_{t}+d_{t}
\end{array}\right\}
$$

Where; $x_{t}$ is the state vector; $u_{t}$ is the manipulated input variables; $d_{t}$ is the disturbance; the measured outputs $y$ which satisfies the objective function and the controlled outputs $z_{t}$; and $A, B$ and $C$ are control parameters.

Second step the performance index which is the objective function that will be optimized. Where; in this step, the difference between the output and the reference signal is computed using Eq. 11 then MPC trying to reduce the error:

$$
\varepsilon(t+k \mid t)=\hat{y}(t+k \mid t)-r(t+k \mid t)
$$

$\left.J(v, t)=\sum_{k=0}^{N-1} \varepsilon^{\top}(t+k \mid t) \Gamma(k) \varepsilon(t+k \mid t)\right\}$

Where: $\varepsilon(t+k \mid t)$ is the predicted error; $\hat{y}(t+k \mid t)$ is the predicted value; $J(v, t)$ is the system performance index; $\Gamma(k)$ is the degree of importance; and $N$ is time steps till horizon.

The third step is constraint wherein this step the output signal is subject to restrictions such as the following equations:

$$
\begin{gathered}
u_{k, \min }<u_{k}<u_{k, \max } \\
y_{k, \min }<y_{k}<y_{k, \max } \\
\Delta(t+k \mid t)=0 \quad \forall k \geq N_{c}
\end{gathered}
$$

The fourth step is optimization where optimize technique is applied to compute a sequence of future control trajectory that minimizes the performance index subject to the given constraints.

The final step is repeating where MPC implements the first step of the optimal input signal until new measurements are available.

\section{Case Study}

In this paper, a case study is designed to study the power flow with the DLR effect in grids with high penetration of renewable energy "wind and PV". The system is the 14bus system that represents the south portion of the Egyptian grid as shown if Fig. 5. This system consists of six generators plus Zafarana and Gabal-Zeet wind farm, and Benban solar park. The wind farm has a capacity of $3500 \mathrm{MW}$ and connects to Cairo by $220 \mathrm{KV}$ OHTL with a length of $200 \mathrm{Km}$, while solar capacity is $2000 \mathrm{MW}$ and connects to Cairo by $500 \mathrm{KV}$ OHTL with a length of 900 $\mathrm{Km}$. The system data were collected for the period from May 2018 to Oct 2019, this data was collected from the Egyptian grid [12]. The available data enabled good training for the proposed model.

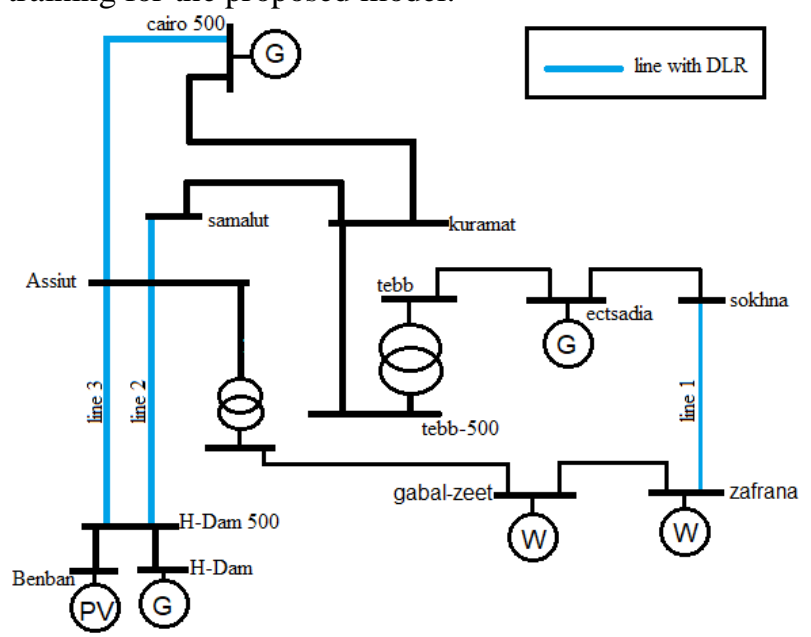

Fig. 5: south portion of the Egyptian grid

In this research, DLR is studied on three lines, these line data are shown in the following table.

\begin{tabular}{|c|c|c|c|}
\hline Line & Voltage $(\mathrm{KV})$ & Length $(\mathrm{Km})$ & Capacity (MVA) \\
\hline Line 1 & 220 & 200 & 600 \\
\hline Line 2 & 500 & 550 & 1200 \\
\hline Line 3 & 500 & 900 & 1200 \\
\hline
\end{tabular}

The proposed power flow model using MPC based on DLR has applied for one day 10 Aug 2019. This day has a high temperature which has significant effect on DLR; therefore this day is one of the best days to study the proposed model. The power generated by the stations whose energy will be transferred over the three proposed lines is shown in Fig. 6.

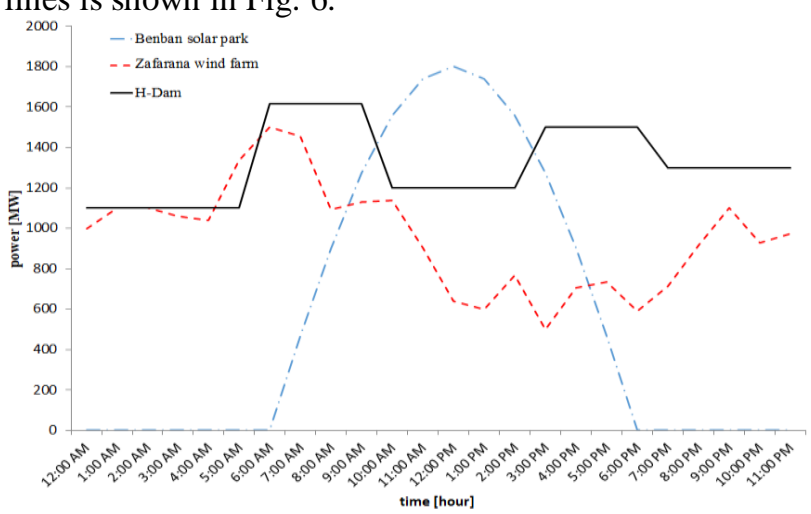

Fig. 6: Power generation

Fig. 7 and Fig. 8 demonstrated DLR, SLR, and the actual power transferred through the lines. Fig. 7 presented the power flow in line 1. Also, Fig 8 presented the power flow in line 2 and line 3 , they are parallel transferring the same 
power and facing the same meteorological conditions. Line 1 is approximately short compared to lines 2 and 3 . There is no significant temperature changes along the line 1 as it is mainly located on the same longitude limiting to some extent the ambient temperature effect. A new challenge arises for lines 2 and 3 as there DLR is not constant throughout the entire line due to the temperature changes as they connect northern and southern parts of Egypt. This problem mandates that we keep the DLR constant at the local value of the DLR at the critical span.

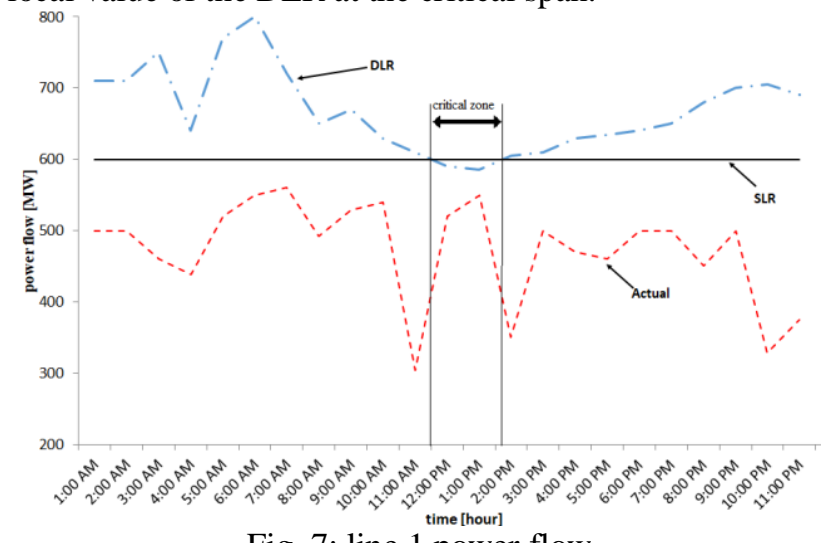

Fig. 7: line 1 power flow

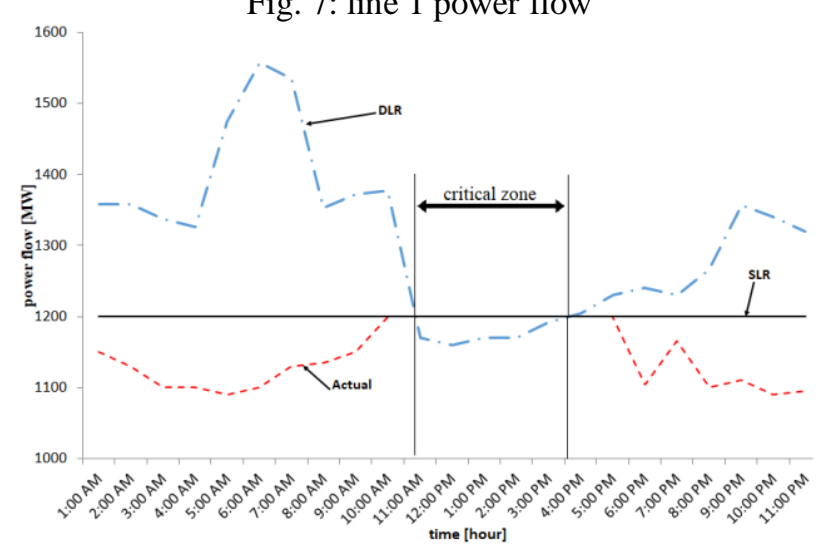

Fig. 8: line 2 and line 3 power flow

From Fig. 7 and Fig. 8, it is noticed that the power flow in the OHTL using the DLR concept is more than the actual power flow. This leads to increase the power supply from the Zafarana wind farm to the electrical grid by $10 \%$ and increase the supplied energy from $\mathrm{H}$-dam and Benban solar park by $9.6 \%$. Also, it is noticed that in the period between 11:30 AM to 2:10 PM at Fig. 7 and from 11:15 AM to 4:00 PM in Fig. 8, the DLR amount is lower than the SLR according to increasing the atmospheric temperature. Therefore, the control model must reduce the power flow in these intervals to improving the system reliability. This does not happen when the operator is using SLR technique.

Finally, the proposed improved power flow with DLR effect using MPC enables the operating system to increased line ratings on existing and new transmission lines, reduced need for special protection schemes, and reduced carbon footprint.

\section{Conclusion}

This paper discussed Dynamic Line Rating (DLR) and its use for enhancing the power flow in the system. An automotive control technique to manage the power flow in the overhead transmission line (OHTL) by using a combination of a control technique using model predictive control (MPC) and prediction concept using the artificial neural network (ANN). ANN is used to predict DLR as a function of environmental impact. Mathematical models and applications to one-year data are presented in the paper. The system under study in this paper is a portion of the Egyptian grid supplied from a hybrid renewable energy.

\section{References}

1. Bucher, M.A. and G. Andersson, Robust corrective control measures in power systems with dynamic line rating. IEEE Transactions on Power Systems, 2015. 31(3): p. 2034-2043.

2. Talpur, S., et al., Dynamic line rating for wind power. Journal of Renewable and Sustainable Energy, 2016. 8(1): p. 013114.

3. Naim, W., K. Morozovska, and P. Hilber, Effects of Dynamic Line Rating on the Durability and Mechanical Strength of Aluminum Cable Steel Reinforced (ACSR) Conductors. Energy Procedia, 2019. 158: p. 3164-3169.

4. Ngoko, B.O., H. Sugihara, and T. Funaki, Effect of Dynamic Line Ratings on Optimal Dispatch Considering Uncertainty Costs due to Intermittent Renewable Energy. IFACPapersOnLine, 2018. 51(28): p. 185-190.

5. Uski, S., Estimation method for dynamic line rating potential and economic benefits. International Journal of Electrical Power \& Energy Systems, 2015. 65: p. 76-82.

6. Aznarte, J.L. and N. Siebert, Dynamic line rating using numerical weather predictions and machine learning: A case study. IEEE Transactions on Power Delivery, 2016. 32(1): p. 335-343.

7. Karimi, S., P. Musilek, and A.M. Knight, Dynamic thermal rating of transmission lines: A review. Renewable and Sustainable Energy Reviews, 2018. 91: p. 600-612.

8. Jin, L., R. Kumar, and N. Elia, Model predictive control-based real-time power system protection schemes. IEEE Transactions on Power Systems, 2009. 25(2): p. 988-998.

9. Almassalkhi, M. and I. Hiskens. Temperaturebased model-predictive cascade mitigation in electric power systems. in 52nd IEEE Conference on Decision and Control. 2013. IEEE.

10. Michiorri, A., et al., Forecasting for dynamic line rating. Renewable and sustainable energy reviews, 2015. 52: p. 1713-1730.

11. Megahed, T.F., S.M. Abdelkader, and A. Zakaria, Energy Management in Zero-Energy Building using Neural Network Predictive Control. IEEE Internet of Things Journal, 2019.

12. Egyptian New and Renewable Energy Association, NREA Annual Report, 2018, URL: http://www.nrea.gov.eg/annual-english-2018-2019.pdf. 